\title{
Exact renormalization group with optimal scale and its application to cosmology
}

\author{
Benjamin Koch and Israel Ramirez \\ Pontificia Universidad Católica de Chile, \\ Av. Vicuña Mackenna 4860, \\ Santiago, Chile
}

\begin{abstract}
Assuming an effective gravitational action with scale dependent coupling constants, a consistency condition for the local form of the cut-off scale is derived. The approach is applied to homogeneous cosmology and running couplings with an ultraviolet fixed point. Within the given approach this allows to derive bounds on the value of the fixed point.
\end{abstract}

PACS numbers: 04.62.+v, 03.65.Ta 


\section{Introduction}

Finding a consistent and predictive description of quatum gravity is a long standing problem. Aside from the most popular approaches such as loop quantum gravity, the technique of Exact Renormalization Groups (ERG) has recently attracted more interest. This technique allows to derive equations for the running of couplings of an effective average action without any power expansion in the couplings. It has been conjectured that the non-renormalizability of gravity is actually an artefact of the loop expansion in the gravitational coupling and that in an exact calculation the (dimensionless) couplings actually run to non trivial ultra violet fixed points. Since this running would countervail the divergencies it is called asymptotic safety scenario [1. In several analytical and numerical studies supporting evidence for this kind of scenario has been found [2, 3, 4, [5, 6, 7, 8, 9]. In order to test the implications of this approach it has been studied in a large variety of contexts: Cosmology and Astrophysics [10, 11, 12, 13, 14, 15, 16], Brans Dicke theory [17], black holes [18, 19, 20, 21, 22], black holes in extra dimensions [23, 24, 25, 26], interactions in extra dimensions [6], modified dispersion relations [27], $\mathrm{f}(\mathrm{R})$ gravity [28], deformed special relativity [29], and gravitational collapse [30]. This work contributes to the above studies in three ways:

In section 2 a simple expansion is used in order to derive a compact analytic form for the running couplings $G_{k}$ and $\Lambda_{k}$ with two fixed point parameters $g^{*}$ and $\lambda^{*}$.

In section 3 a new approach for the choice of the cut-off scale $k$ is proposed. In the suggested choice, all solutions respect diffeomorphism invariance by construction. This feature is the main difference from existing studies.

In section 4 the findings from the sections 2 and 3 are applied to cosmology and conditions on the fixed point parameters $g^{*}, \lambda^{*}$ are derived and discussed.

\section{An Approximate Analytical Solution to Exact Renormalization Group Equations}

The Exact Renormalization Group (ERG) was studied in the context of effective potentials [31]. This lead to an Exact Renormalization Group Equation (ERGE)

$$
\partial_{t} \Gamma_{k}=\frac{1}{2} \operatorname{Tr}\left(\Gamma_{k}^{(2)}+R^{(0)}\right)^{-1} \partial_{t} R^{(0)}
$$

where $\Gamma_{k}$ stands for the effective average action, $R^{(0)}$ is the momentum-cut-off, and $\operatorname{Tr}$ stands for the sum over all indices in DeWitt notation (see also [33] for a recent review). For the case of quantum gravity in the Einstein-Hilbert tuncation the effective action in four dimensions is given by,

$$
\Gamma_{k}=\int d^{4} x \frac{\sqrt{g}}{16 \pi G_{k}}\left[R(g)-2 \Lambda_{k}\right],
$$

where the subscript $k$ denotes that $G$ and $\Lambda$ are scale-dependent functions [4]. For the ERGE equations (1) and the effective action (2) one defines the dimensionless 
renormalized gravitational and cosmological constants,

$$
g_{k}=k^{2} G_{k} \quad, \quad \lambda_{k}=\frac{\Lambda_{k}}{k^{2}} .
$$

Using a sharp cut-off [5] the beta functions for those constants are found to be

$$
\begin{aligned}
& \beta_{\lambda}=\partial_{t} \lambda_{k}=\frac{P_{1}}{P_{2}+4\left(4+2 g_{k}\right)}, \\
& \beta_{g}=\partial_{t} g_{k}=\frac{2 g_{k} P_{2}}{P_{2}+4\left(4+2 g_{k}\right)},
\end{aligned}
$$

where $t=\ln k$ and $P_{i}$ are polynomials of $g_{k}$ and $\lambda_{k}$ [4, 19]. Those equations have the approximate analytical solution of the form

$$
\begin{aligned}
g(k) & =\frac{k^{2}}{1+k^{2} / g^{*}}, \\
\lambda(g) & =\frac{g^{*} \lambda^{*}}{g}\left((5+e)\left[1-g / g^{*}\right]^{3 / 2}-5+3 g /\left(2 g^{*}\right)\left(5-g / g^{*}\right)\right),
\end{aligned}
$$

which directly defines $\lambda(k)$. While the functional form of $g(k)$ is frequently used, $\lambda(g)$ is a new approximation, which is the result of a Taylor expansion around $\lambda \ll 1$. The

constant $e$ arises as integration constant of the equations (44(5). As shown in the following study on cosmology this constant can be fixed from the infrared behavior of $\lambda_{k}$ and $g_{k}$. The constants $\lambda^{*}$ and $g^{*}$ are parameterizations of the UV fixed points

$$
g\left(k^{2} \rightarrow \infty\right)=g^{*} \text { and } \lambda\left(k^{2} \rightarrow \infty\right)=\lambda^{*} .
$$

Numerical results [6, 7], reveal evidence for specific values for the fixed point, in this work however $\lambda^{*}$ and $g^{*}$ will be treated as free parameters, that should be restricted or determined by observational data. Figure 1 shows the behavior of the numeric and the analytic solutions (6), 7). The numerical solution in figure $1 \mathrm{1b}$ is however limited to the curves on the left of the parabula, where the beta functions (4, 5) become infinite.

Although, those results are obtained in spaces with Euclidian signature $(+,+,+,+)$, they will also be used in metric spaces with the signature $(-,+,+,+)$.

\section{A consistent cut-off scale}

Coupling the Einstein-Hilbert action to matter with scale dependent couplings $\Lambda_{k}$ and $G_{k}$ gives

$$
S[g]=\int d^{4} x \sqrt{-g}\left(\frac{R-2 \Lambda_{k}}{16 \pi G_{k}}+\mathcal{L}_{m}\right) .
$$

The equations of motion for the metric field in (91) are

$$
G_{\mu \nu}=-g_{\mu \nu} \Lambda_{k}+8 \pi G_{k} T_{\mu \nu}-\Delta t_{\mu \nu} \quad,
$$

where the possible coordinate dependence of $G_{k}$ induces an additional contribution to the stress energy tensor [12, 32]

$$
\Delta t_{\mu \nu}=G_{k}\left(g_{\mu \nu} \square-\nabla_{\mu} \nabla_{\nu}\right) \frac{1}{G_{k}} .
$$




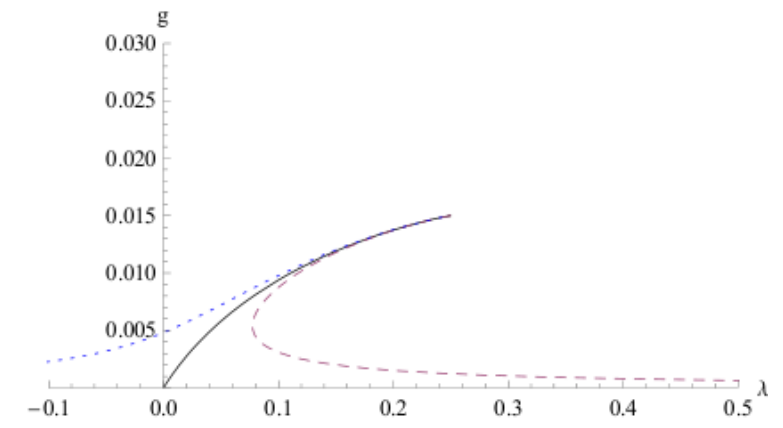

(a)

Analytical solution to the approximated ERGEs for the values $g^{*}=0.016$ and $\lambda^{*}=0.25$. Trajectories with $e>0$ imply that $\lambda>0$, trajectories with $e=0$ imply that $\lambda \rightarrow 0$ in the $\lambda$-g infrared, and trajectories with $e<0$ give $\lambda>0$ in the ultra violet and $\lambda<0$ in the infrared.

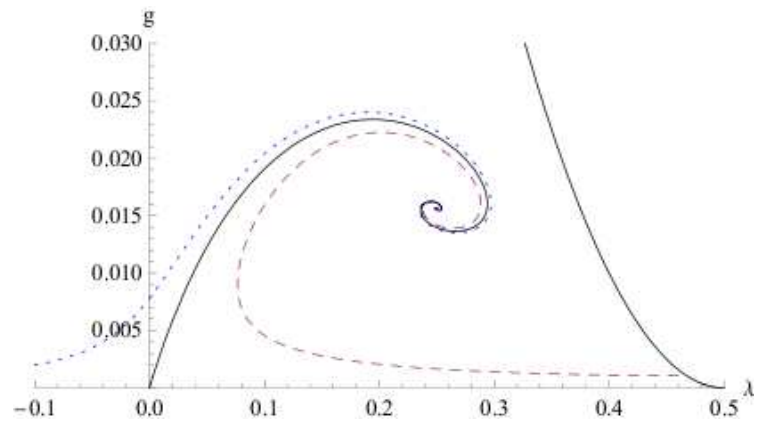

(b)

Figure 1: Solutions to the ERGEs (4, 5). for $G_{0}=1$.

The equations of motion (10) and their solution depend crucially on the choice of the cut-off scale $k$. In particle physics such a scale would be chosen according to the energy of the process that is examined. In a curved spacetime such a definition has no global unique meaning and the choice of the local functional form of $k\left(x^{\mu}\right)$ becomes tricky if not even arbitrary. As explained in [34] $k\left(x^{\mu}\right)$ has to be guessed for each specific problem. Typically $k \sim 1 /\left(\int d s\right)$ is used for black holes [19, 25] and $k \sim 1 / t$ or $k \sim 1 / H$ is used for cosmology [11, 16]. Any such choice, however, implies further difficulties: General covariance dictates that the equations of motion have to fulfill the Bianchi identities

$$
G_{\mu \nu} ; \nu=g_{\mu_{\nu}} ; \nu=0 \quad .
$$

We will assume that the conservation of the matter stress energy tensor persists

$$
T_{\mu \nu} ; \nu=0 .
$$

Although this assumption is plausible, it might not be generally true, for example it does not hold whenever particles are created (as it is presumable the case for non extremal black holes). By using (13) and (12) it follows for the right hand side of (10) that

$$
\left(8 \pi G_{\bar{k}}^{\prime} T_{\mu \nu}-g_{\mu \nu} \Lambda_{\bar{k}}^{\prime}\right) \partial^{\nu} k-\nabla^{\nu} \Delta t_{\mu \nu}=0,
$$

where $\partial_{\mu} G_{k}=\left(\partial G_{k} / \partial k\right)\left(\partial_{\mu} k\right)=G_{k}^{\prime} \partial_{\mu} k$ was applied. In most existing studies [10, 11, 12, 12, due the explicit choice of the functional form of $k(x)$, one always had to sacrifice either general covariance (12), or the conservation of the stress energy tensor (13). In contrast to this, we propose to use the identity (14) in order to determine the relation between $k$ and $x^{\nu}$. Thus, by choosing $k(x)$ to be a solution of the consistency equation (14), general covariance and conservation of the stress-energy tensor can be perserved by construction. This procedure is analog to the one that was discussed in [35, 36], here however also the induced stress-energy tensor $\Delta t_{\mu \nu}$ is taken into account. 
In the spirit of [37] it would also be desirable to have a physical result which does not depend on the local choice of the cut-off scale $k$. This can be achieved by treating the function $k$ like a classical field and minimizing the effective action (9) with respect to this field. The corresponding equation of motion for $k$ is

$$
2 \frac{\Lambda_{k} G_{k}^{\prime}}{G_{k}^{2}}-R \frac{G_{k}^{\prime}}{G_{k}^{2}}-2 \frac{\Lambda_{k}^{\prime}}{G_{k}}=0
$$

Although the "equation of motion" for $k^{2}$ (15) and the consistency condition (14) were derived from completely different motivations, they are equivalent. This equivalence can be shown by using (12) and the identity

$$
\nabla^{\nu}\left(\nabla_{\mu} \nabla_{\nu}-g_{\mu \nu} \square\right)\left(\frac{1}{G_{k}}\right)=R_{\nu \mu} \nabla^{\nu}\left(\frac{1}{G_{k}}\right),
$$

which allows to rewrite the consistency condition (14) in the form

$$
R \nabla_{\mu}\left(\frac{1}{G_{k}}\right)-2 \nabla_{\mu}\left(\frac{\Lambda_{k}}{G_{k}}\right)=0 .
$$

The above relation is equivalent to (15), since the only $x_{\mu}$ dependence in the couplings comes due to the functional form of $k^{2}$. This can be seen by explicitly writing out the covariant derivatives in (17). Although a minimal dependence of $k$ and a consistency of the equations of motion are desirable, it is not clear whether they corresponds to a well defined limit in the framework of the exact renormalization group equation (10). This issue arises since in this limit the effective average action becomes extremal and the derivation of the ERGE as it is performed in [4] might not be valid any more. However, in this work it will be supposed that the solutions (6, 7) are still valid.

\section{Cosmology}

In this section the concepts that were introduced in the previous sections will be applied to spatially homogeneous universe, which can be parameterized by the metric

$$
d s^{2}=-d t^{2}+a(t)^{2} d \vec{x}^{2}
$$

Like in the case of standard cosmology the matter stress energy tensor will be assumed to be a perfect fluid. The homogeneity should also hold for the running coupling constants and the scale parameter $k=k(t)$. Having time dependencies only one finds that the induced stress energy tensor takes the form

$$
\begin{aligned}
\Delta t_{00} & =-\frac{3 \dot{G}_{k} \dot{a}}{G_{k} a} \\
\Delta t_{i i} & =\frac{a}{G_{k}^{2}}\left(a \ddot{G}_{k} G_{k}-2 \dot{G}_{k}^{2} a+2 \dot{G}_{k} \dot{a} G_{k}\right) \\
\Delta t_{\mu \neq \nu} & =0 .
\end{aligned}
$$

With this the Einstein equations reduce to generalizations of the Friedmann equations

$$
\begin{aligned}
& \left(\frac{\dot{a}}{a}\right)^{2}=\frac{8 \pi G_{k}}{3}\left(\frac{a_{0}^{4} \rho_{r}}{a^{4}}+\frac{a_{0}^{3} \rho_{m}}{a^{3}}\right)+\frac{\Lambda_{k}}{3}-\frac{\kappa}{a^{2}}+\frac{\dot{G}_{k} \dot{a}}{G_{k} a}, \\
& \frac{\ddot{a}}{a}=-\frac{8 \pi G_{k}}{3}\left(\frac{a_{0}^{4} \rho_{r}}{a^{4}}+\frac{a_{0}^{3} \rho_{m}}{2 a^{3}}\right)+\frac{\Lambda_{k}}{3}+\frac{\dot{G}_{k} \dot{a}}{2 G_{k} a}+\frac{G_{k} \ddot{G}_{k}-2 \dot{G}_{k}^{2}}{2 G_{k}^{2}} .
\end{aligned}
$$


The two equations (20, 21) would be equivalent for a fixed coupling $\dot{G}_{k}=0$, but they are not equivalent for the time dependent coupling $\dot{G}_{k} \neq 0$. Without abandoning the conservation of energy and momentum this problem can be solved if both $G_{k}$ and $\Lambda_{k}$ are time dependent quantities [38, 39]. In the context of ERGE it means that the consistency condition (14) has to be imposed. For the above equations one finds

$$
8 \pi \dot{G}_{k}\left(\frac{a_{0}^{4} \rho_{r}}{a^{4}}+\frac{a_{0}^{3} \rho_{m}}{a^{3}}\right)+\dot{\Lambda}_{k}+3 \dot{G}_{k} \frac{\dot{a} \dot{G}_{k}+G_{k} \ddot{a}}{a G_{k}^{2}}=0 .
$$

One can show that this condition makes the two equations (20, 21) mathematically equivalent and thus it is sufficient just to work with (20) and (22). By the use of equation (20), the matter part in (22) can be replaced leading to a more compact form of the consistency condition

$$
3 \alpha(t)-\Lambda_{k}+G_{k} \frac{\dot{\Lambda}_{k}}{\dot{G}_{k}}=0,
$$

where we defined $\alpha(t)=(\dot{a} / a)^{2}+\ddot{a} / a+\kappa / a^{2}$. Thus, the problem can in principle be reduced to first solving (23) in order to find the functional relation for $k=k(\alpha)$ and then inserting this into the first Friedman equation (20), which then has to be solved analytically or numerically.

Before starting with this procedure it is interesting to return for a moment to the equation which was obtained from demanding a minimal scale dependence of the effective action. For the homogeneous metric (18) this equation (15) is exactly the same as the consistency condition (23). This identity is not a coincidence but rather comes from a deeper connection between (14) and (15) that was generally shown at the end of the previous section.

For finding solutions of the system (23, 20) an explicit form of the running couplings $\Lambda_{k}$ and $G_{k}$ has to be assumed. In order to maintain analytical feasibility of the problem the approximated solutions (6, (7) will be used. The first task is to solve (23) in

order to find the functional form of $k=k(\alpha)$. The solution is however a relatively large expression, which is not instructive in this form. Furthermore, this algebraic expression has to be inserted into the generalized Friedman equation (20). This gives a complicated non-linear differential equation of higher order, which can probably not be solved analytically. Therefore, the model will be studied in the infrared (IR) and the ultraviolet (UV) limit.

\subsection{ERG cosmology in the IR}

In this limit it is assumed that the energy scale is way below the Planck scale $k^{2} \ll 1 / G_{0}$. This allows to expand (23) in a Taylor series around $k^{2}=0$

$$
3 \alpha+\frac{k^{2} \lambda^{*}}{4}(-3+e)+\frac{e \lambda^{*} g^{*}}{2 G_{0}}=0+\mathcal{O}\left(k^{4} G_{0}^{2}\right) .
$$

which is solved by $k_{I R}^{2}=\left(2 e \lambda^{*} g^{*}-12 G_{0} \alpha\right) /\left((-3+e) G_{0} \lambda^{*}\right)$. Since the constants $\lambda^{*}, g^{*}, G_{0}$ are positive one sees that for $e<3$ the numerator of this solution has to be less than 
zero for the square of the energy scale $k_{I R}^{2}$ to be positive. For decreasing $\alpha$ this does not hold any more as soon as $\alpha \leq e \lambda^{*} g^{*} /\left(6 G_{0}\right)$. The solution to this problem is that from this moment on the IR limit is reached and the running of the couplings stops. Thus the infrared solution for $k(\alpha)$ reads

$$
k_{I R}^{2}=\left\{\begin{array}{cc}
\frac{2 e \lambda^{*} g^{*}-12 G_{0} \alpha}{(-3+e) G_{0} 2 \lambda^{*}} & \text { for } \alpha \geq e \lambda^{*} g^{*} /\left(6 G_{0}\right) \\
0 & \text { for } \alpha<e \lambda^{*} g^{*} /\left(6 G_{0}\right)
\end{array} .\right.
$$

Given the typical values for $g^{*}$ and $\lambda^{*}$ [4, 5] of order one, todays value of $\alpha$ definitely demands that $k_{\text {today }}^{2}=0$. In this limit the modified Friedmann equation (20) takes the familiar form

$$
\left(\frac{\dot{a}}{a}\right)^{2}=\frac{8 \pi G_{0}}{3}\left(\frac{a_{0}^{4} \rho_{r}^{0}}{a^{4}}+\frac{a_{0}^{3} \rho_{m}^{0}}{a^{3}}\right)+\frac{e g^{*} \lambda^{*}}{3 G_{0}}-\frac{\kappa}{a^{2}} .
$$

This construction is necessary in order to avoid problems of the big rip type [40]. For the model to agree with reality, this equation has to be the same as the standard Friedmann equation [41]. Enforcing this identity one finds that the initially arbitrary integration constant $e$ of the exact renormalization group equations has to be adjusted to the observed value of the cosmological constant

$$
e=\Lambda_{\text {observed }} \cdot \frac{G_{0}}{g^{*} \lambda^{*}} .
$$

This is a very strong constraint since it reduces the infinite set of trajectories that solve (11) to one single curve only. Within error bars this curve is characterized by the positive value given in (27). This corresponds to a trajectory in the figure 1 where $\lambda$ is positive for all values of $k$. As suggested here, the transition between the cosmology with a variable scale (20) and the IR limit (26) is not smooth. If one sticks to the approximated analytical form of the couplings as shown in figure 1a it implies violation of total energy and momentum. This is due to the fact, that the last term of eq. (20) still is non zero at the moment of transition to $k\left(\alpha_{0}\right)=0$. A straight forward way out of the dilemma is to conjecture that this additional energy $\delta E$ is transferred to the matter and radiation content of the universe such that

$$
\rho_{r} \rightarrow \rho_{r}^{0}=\rho_{r}+\delta \rho_{r} \quad \text { and } \quad \rho_{m} \rightarrow \rho_{m}^{0}=\rho_{m}+\delta \rho_{m} \quad .
$$

Where $\rho_{x}$ is the density before the transition and $\rho_{x}^{0}$ is the density after the transition. Such a process can be seen as the analog of reheating in standard cosmology. The value of the transition energy will be calculated in the discussion section. There is however numerical evidence for the curves in figure $1 \mathrm{~b}$ that $\lim _{\alpha \rightarrow 0} k^{2}=0$, which would make the issue of negative $k^{2}$ disappear.

\subsection{ERG cosmology in the $U V$}

The very early universe was presumably a very hot environment. Therefore one expects that good estimates for the Planckian and pre-Planckian epoch can be obtained by an expansion in $\left(1 /\left(k^{2} G_{0}\right) \ll 1\right)$. In this limit the consistency condition (23) reads

$$
3 \alpha-k_{U V}^{2} 2 \lambda^{*}+\frac{10 \lambda^{*} g^{*}}{4 G_{0}}=0+\mathcal{O}\left(1 /\left(k^{4} G_{0}^{2}\right)\right) .
$$


There is no maximal value for the scale $k^{2}$ and thus the asymptotic solution of (23) is

$$
k_{U V}^{2}=\frac{3 \alpha}{2 \lambda^{*}} \text {. }
$$

Inserting this solution back into the generalization of the first Friedman equation still gives a non-linear differential equation of third order, which can not be trivially solved. However, for asymptotically small times a solution can be found by making the linear ansatz

$$
a=C \cdot t
$$

where $C$ is a constant that still has to be determined. With the relation (30) and the ansatz (31) the differential equation (20) reduces to

$$
\frac{1}{t^{2}}=\frac{45 C^{4}+a_{0}^{4} \pi \rho_{r} \lambda^{*} 32 g^{*}-9 \kappa}{18 C^{2} t^{2}\left(C^{2}+\kappa\right)}+\mathcal{O}\left(t^{0}\right)
$$

This proves that (31) is a solution of the generalized Friedman equation and that the only possibly positive linear expansion coefficient is

$$
C=\frac{1}{3} \sqrt{-3 \kappa+2 \sqrt{-24 a_{0}^{4} \pi \rho_{r} \lambda^{*} g^{*}+9 \kappa^{2}}} .
$$

This, implies that this model only has a physical solution in the UV if the curvature is different than zero and fulfills

$$
\begin{aligned}
& \kappa>\frac{4}{3} a_{0}^{2} \sqrt{2 \pi \rho_{r} \lambda^{*} g^{*}} \quad \text { or } \\
& \kappa<-2 a_{0}^{2} \sqrt{\frac{2}{3} \pi \rho_{r} \lambda^{*} g^{*}} .
\end{aligned}
$$

The above inequalities can be translated to dimensionless matter parameters

$$
\Omega_{r}=\frac{8 \pi G_{0}}{3 H_{0}^{2}} a_{0}^{4} \rho_{r}, \quad \Omega_{k}=-\frac{\kappa}{H_{0}^{2}}, \quad \Omega_{\Lambda}=\frac{\Lambda}{3 H_{0}^{2}}
$$

Thus, the relation (34) restricts the remaining parameters of the running couplings

$$
\frac{3}{4} G_{0} H_{0}^{2} \frac{\Omega_{k}^{2}}{\Omega_{r}}>\lambda^{*} g^{*}
$$

where it is important to remember, that due to the energy transfer (28), the value of $\Omega_{r}$ is not necessarily the same as the one observed today $\Omega_{r}^{0}$.

\section{Discussion}

The behavior of the solution of the condition (23) can best be demonstrated graphically. In figure 2, the dependence between the dimensionless quantities $G_{0} k^{2}$ and $G_{0} \alpha$ is shown. The IR limit corresponds to the left region of the figure 2 . One sees that it shows no running of the couplings at the value $k^{2}=0$, and a linear dependence between $G_{0} k^{2}$ and $G_{0} \alpha$ for small values of $G_{0} k^{2}$. The UV limit corresponds to the far right hand side of the plot, with large values of $G_{0} k^{2}$. In this limit the linear growth continues as it is already visible on the right hand side of the figure, which reflects directly the findings of the approximation (30). 


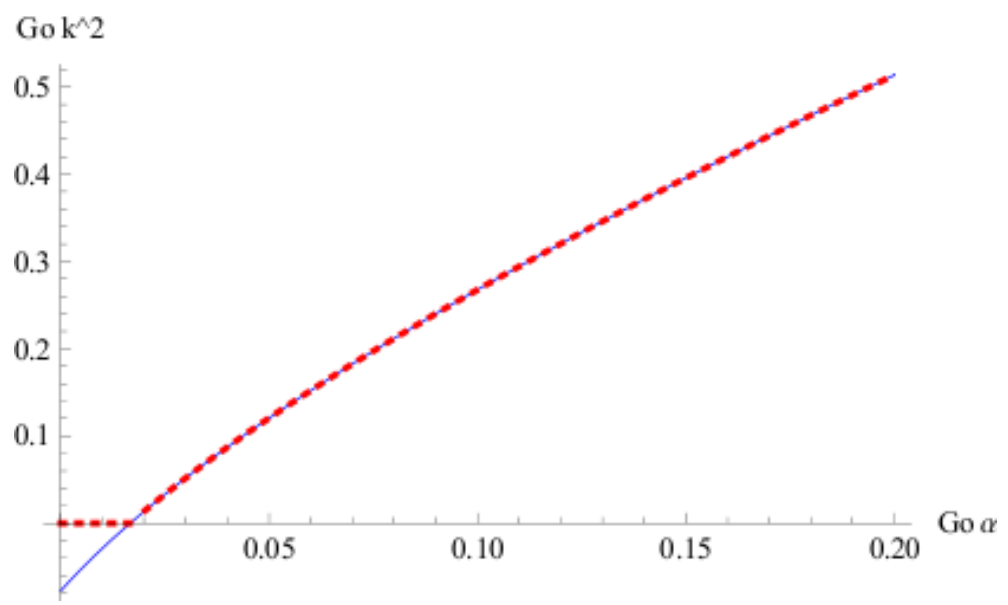

Figure 2: Functional dependence of $G_{0} k^{2}$ and $G_{0} \alpha$ for $e=0.1$. The blue line is the complete solution of the condition (23), while the red-dotted line includes the condition (25) in the infrared limit.

As explained in section 4.2, this UV dependence implies an epoch of linear expansion in the early universe (31). A result which offers a potential explanation to the homogeneity of the cosmic microwave background, alternative to inflation. This is due to the fact that in a linearly expanding universe the causal horizon scales as

$$
h_{c}=\int_{t_{i}}^{t_{f}} d t \frac{c}{a(t)}=\frac{c}{C}\left[\ln \left(\frac{t_{f}}{t_{i}}\right)\right] .
$$

In contrast to this, the Hubble Horizon in this epoch scales as

$$
h_{H}=\frac{1}{t_{f}-t_{i}} \int_{t_{f}}^{t^{i}} \frac{c}{\dot{a}}=\frac{c}{C} .
$$

Therefore, the early epoch of linear expansion can create arbitrarily high homogeneities for $t_{i} \rightarrow 0$.

Although this is a nice feature of the model, the drawbacks become obvious as soon as one inserts numbers into the relation (36). Using the given limits on $\Omega_{k}$ [42] it reveals that the product of the fixed point parameters $\lambda^{*}$ and $g^{*}$ has to be of the order of $G_{0} H_{0}^{2} \approx 10^{-122}$, if the original radiation density $\Omega_{r}$ was of the same order of magnitude as it is today! When working with the analytical solutions one has to study whether one can evade this extreme bound by assuming that $\Omega_{r}$ was extremely small, and all of the radiation density observed today $\Omega_{r}^{0}$ was produced during the transition (25) from running couplings to constant couplings at $k^{2}=0$. However, estimating the last term in (20) in a linear expansion solution like (31) and comparing it at the moment of transition to the radiation radiation term in (26) in the same solution gives

$$
\lambda^{*} g^{*} \approx \frac{64}{3(3-e)} G_{0} H_{0}^{2} \frac{1}{\Omega_{r}^{0}} .
$$

Thus, evidently, the idea that the observed radiation density is due to the IR transition $\Omega_{r}^{0} \approx \delta \Omega_{r}$ also implies values of the parameters $g^{*} \lambda^{*} \approx 10^{-120}$. One last backdoor 
might lie in the possibility that $e \approx 3$ and the numerator of (39) is going to zero. But remembering the relation (27) this possibility implies

$$
\lambda^{*} g^{*} \approx G_{0} H_{0}^{2} \Omega_{\Lambda} .
$$

Like before this means that $g^{*} \lambda^{*} \approx 10^{-122}$. Thus, in all possible scenarios one obtains that the presented model only is in agreement with observational data in cosmology if the parameters $g^{*} \cdot \lambda^{*}$ are extremely small $\left(\approx 10^{-122}\right)$.

\section{Conclusion}

A new approach for finding the optimal and consistent cut off scale $k^{2}$ for EinsteinHilbert action is presented. This is achieved by taking the relation (15) as algebraic condition for the local functional form of the scale $k^{2}(x)$. In this context it has been shown that (15), which reflects a minimal $k^{2}$ dependence as suggested by Weinberg [37], implies the consistency condition (14) and vice versa. An elegant way of expressing this condition is given by eq. (17)

$$
R \nabla_{\mu}\left(\frac{1}{G_{k}}\right)-2 \nabla_{\mu}\left(\frac{\Lambda_{k}}{G_{k}}\right)=0
$$

In order to test the new framework it is applied to cosmology for the case of the approximate analytic ERG solutions (6),7). The cosmological model obtained by this procedure offers some promising properties such as standard Friedmann cosmology in the IR and a linear expansion in the UV. However, it is also found that the parameters of the UV fixed point have to take take extreme values $g^{*} \lambda^{*} \approx 10^{-120}$ for the model to be in agreement with observed cosmological parameters. The order of magnitude of the parameters reminds of the vacuum density problem in quantum field theory. Such a small value for the fixed point parameters does not only appear to be extremely fine tuned, it is also in disagreement with the findings from approximate numerical fixed point searches [6, 7, 28, 9]. Thus, demanding to have a classically consistent effective action (with demanding (13)) for running dimensionless couplings (6. 17) with a fixed point at the order of 0.1 does not produce a good cosmological model. This allows for at least three different types of conclusions:

- First, the findings could simply mean that a more complete cosmological model including other fields (quintessence, inflaton, ...) is needed. This possibility can be checked by trying more general models, but it is not very attractive, since one has to introduce new parameters which reduces the predictive power of the theory.

- Second, the findings could be a hint that there is a generic problem with the approach. For example it might be necessary to relax the assumption of a conserved stress-energy tensor (13) in the presence of a horizon, where the production of particles can be expected. This possibility should be checked by applying the approach to other problems in general relativity such as black holes.

- Third, the findings could be a consequence of the particular choice that has been made for the functional form of the running couplings $\left(G_{k} \& \Lambda_{k}\right)$. In this context, 
the numerical solution $1 \mathrm{~b}$ should be studied in comparison with the analytical approximation 1a, Since other candidates for effective theories of quantum gravity predict different scaling behavior of the couplings [43, 44, 45], they should also be checked directly within the given approach.

Many thanks to M. A. Diaz, A. Gomberoff, and M. Bañados for helpful hints and discussions. This work was supported by CONICYT project PBCTNRO PSD-73 and FONDECYT project 1090753.

[1] S. Weinberg, General Relativity: An Einstein centenary survey, ed. S. W. Hawking and W. Israel, chapter 16, pp. 790-831; Cambridge University Press.

[2] D. Dou and R. Percacci, Class. Quant. Grav. 15, 3449 (1998) arXiv:hep-th/9707239.

[3] W. Souma, Prog. Theor. Phys. 102, 181 (1999) arXiv:hep-th/9907027.

[4] M. Reuter and F. Saueressig, Phys. Rev. D 65, 065016 (2002) arXiv:hep-th/0110054].

[5] D. F. Litim, Phys. Rev. Lett. 92, 201301 (2004) arXiv:hep-th/0312114.

[6] P. Fischer and D. F. Litim, Phys. Lett. B 638, 497 (2006) arXiv:hep-th/0602203.

[7] D. F. Litim, arXiv:0810.3675 [hep-th].

[8] G. Narain and C. Rahmede, Class. Quant. Grav. 27, 075002 (2010) arXiv:0911.0394 [hep-th]].

[9] K. Groh and F. Saueressig, J. Phys. A 43, 365403 (2010) [arXiv:1001.5032 [hep-th]].

[10] A. Bonanno and M. Reuter, Phys. Lett. B 527, 9 (2002) arXiv:astro-ph/0106468.

[11] A. Bonanno and M. Reuter, Phys. Rev. D 65, 043508 (2002) arXiv:hep-th/0106133.

[12] M. Reuter and H. Weyer, JCAP 0412, 001 (2004) arXiv:hep-th/0410119.

[13] M. Reuter and H. Weyer, Phys. Rev. D 70, 124028 (2004) arXiv:hep-th/0410117.

[14] D. C. Rodrigues, P. S. Letelier and I. L. Shapiro, JCAP 1004, 020 (2010) arXiv:0911.4967 [astroph.CO]].

[15] S. H. Tye and J. Xu, arXiv:1008.4787 [hep-th].

[16] A. Bonanno, A. Contillo and R. Percacci, arXiv:1006.0192 [gr-qc].

[17] M. Reuter and H. Weyer, Phys. Rev. D 69, 104022 (2004) arXiv:hep-th/0311196.

[18] A. Bonanno and M. Reuter, Phys. Rev. D 60, 084011 (1999) arXiv:gr-qc/9811026.

[19] A. Bonanno and M. Reuter, Phys. Rev. D 62, 043008 (2000) arXiv:hep-th/0002196.

[20] K. Falls, D. F. Litim and A. Raghuraman, arXiv:1002.0260 [hep-th].

[21] M. Reuter and E. Tuiran, arXiv:1009.3528 [hep-th].

[22] Y. F. Cai and D. A. Easson, JCAP 1009, 002 (2010) arXiv:1007.1317 [hep-th]].

[23] B. Koch, Phys. Lett. B 663/4, 334 (2008) arXiv:0707.4644 [hep-ph]].

[24] B. Koch, Phys. Lett. B 663, 334 (2008).

[25] T. Burschil and B. Koch, Zh. Eksp. Teor. Fiz. 92, 219 (2010) arXiv:0912.4517 [hep-ph]].

[26] M. Bleicher and P. Nicolini, J. Phys. Conf. Ser. 237, 012008 (2010) arXiv:1001.2211 [hep-ph]].

[27] F. Girelli, S. Liberati, R. Percacci and C. Rahmede, Class. Quant. Grav. 24, 3995 (2007) arXiv:gr-qc/0607030.

[28] A. Codello, R. Percacci and C. Rahmede, Int. J. Mod. Phys. A 23, 143 (2008) arXiv:0705.1769 [hep-th]].

[29] X. Calmet, S. Hossenfelder and R. Percacci, arXiv:1008.3345 [gr-qc].

[30] R. Casadio, S. D. H. Hsu and B. Mirza, arXiv:1008.2768 [gr-qc].

[31] C. Wetterich, Phys. Lett. B 301, 90 (1993).

[32] S. M. Carroll, "Spacetime and geometry: An introduction to general relativity," San Francisco, USA: Addison-Wesley (2004) 513 p.

[33] O. J. Rosten, arXiv:1003.1366 [hep-th].

[34] R. Percacci, arXiv:0709.3851 [hep-th].

[35] A. Babic, B. Guberina, R. Horvat et al., Phys. Rev. D71, 124041 (2005). astro-ph/0407572

[36] S. Domazet, H. Stefancic, arXiv:1010.3585 [gr-qc]].

[37] S. Weinberg, Phys. Rev. D 81, 083535 (2010) arXiv:0911.3165 [hep-th]].

[38] I. L. Shapiro, J. Sola and H. Stefancic, JCAP 0501, 012 (2005) arXiv:hep-ph/0410095. 
[39] J. Sola, J. Phys. A 41, 164066 (2008) arXiv:0710.4151 [hep-th]].

[40] R. R. Caldwell, M. Kamionkowski and N. N. Weinberg, Phys. Rev. Lett. 91, 071301 (2003) arXiv:astro-ph/0302506.

[41] A. Friedman, Gen. Rel. Grav. 31, 122 (1999).

[42] M. Vardanyan, R. Trotta and J. Silk, Mon. Not. Roy. Astron. Soc. 397, 431 (2009) arXiv:0901.3354 [astro-ph.CO]].

[43] P. Nicolini, Int. J. Mod. Phys. A 24, 1229 (2009) [arXiv:0807.1939 [hep-th]].

[44] L. Modesto and P. Nicolini, arXiv:1005.5605 [gr-qc].

[45] R. Garattini and P. Nicolini, arXiv:1006.5418 [gr-qc]. 